\section{La sérotonine prévient l'épilepsie du lobe temporal en inhibant les neurones à bouffées du subiculum}

Anders Victor Petersen, Jean-François Perrier
Département de neurosciences, université de Copenhague, Blegdamsvej 3, 2200 Copenhague, Danemark. perrier@sund.ku.dk

\section{L'épilepsie du lobe temporal}

L'épilepsie du lobe temporal est la forme d'épilepsie la plus fréquente chez I'homme. Cette pathologie chronique récurrente qui concerne trente millions de personnes dans le monde est caractérisée par une hyperactivité neuronale. Les malades sont en général soignés par des drogues qui réduisent l'excitabilité des neurones. Malheureusement, un tiers de ces patients ne répond pas aux traitements pharmacologiques. Le développement de nouvelles stratégies thérapeutiques est par conséquent une priorité pour la recherche biomédicale. Des études effectuées chez des patients ont démontré que les crises d'épilepsie du lobe temporal se déclenchent au sein du subiculum [2]. Les neurones principaux de cette structure sont classés en deux groupes: ceux qui génèrent des potentiels d'action à fréquence régulière et ceux qui déchargent en bouffées (Figure 1). Les bouffées, qui augmentent fortement la probabilité de libération de neurotransmetteurs, sont causées par l'activation du canal calcique membranaire $\mathrm{Ca}_{v} 3$ responsable du courant $T$, caractérisé par un seuil au voltage bas et une ouverture transitoire. 


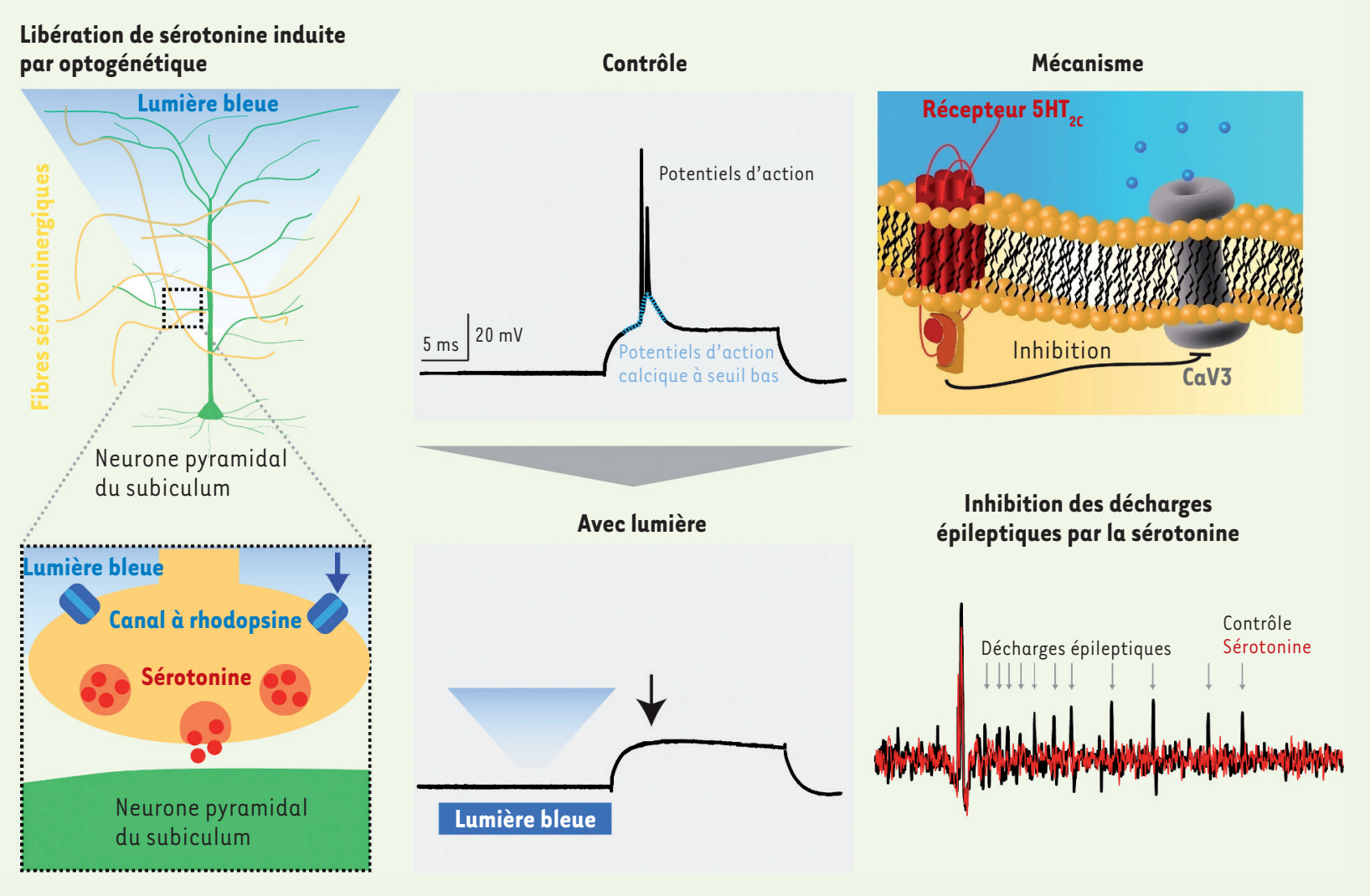

Figure 2. La libération de sérotonine inhibe les bouffées de potentiels d'action et les décharges épileptiques. L'expression sélective du canal à rhodopsine dans les fibres sérotoninergiques permet d'induire, par la lumière bleue, la libération de sérotonine dans la fente synaptique. II en résulte une altération des décharges en bouffées, due à l'inhibition sélective des canaux $\mathrm{Ca}_{v} 3$ (voir la partie mécanisme). L'activation de cette voie réduit fortement la fréquence des décharges épileptiques dans un modèle expérimental, comme cela est indiqué sur le panneau du bas.

Dans les conditions physiologiques, une faible dépolarisation de la membrane est suffisante pour activer ce canal calcique. Il en résulte un influx transitoire de calcium qui génère une dépolarisation brève et déclenche trois ou quatre potentiels d'actions rapprochés les uns des autres correspondant à une bouffée (Figure 1). Le dysfonctionnement de cette propriété intrinsèque peut s'avérer délétère. En effet, les crises d'épilepsie sont initiées par les neurones qui déchargent en bouffées [3, 4], avant de se propager au néocortex. Les crises ont tendance à augmenter l'expression des canaux $\mathrm{Ca}_{v} 3$ et, ainsi, à transformer les neurones à décharge régulière en neurones à bouffées [5], ce qui, à long terme, facilite l'apparition de nouvelles crises. L'inhibition sélective de l'activité électrique des neurones à bouffées apparaît donc comme une piste intéressante pour empêcher le déclenchement des crises d'épilepsie.

\section{La sérotonine inhibe les décharges en bouffées}

Les neurones du subiculum sont richement innervés par des axones qui libèrent de la sérotonine. Lors de notre étude, nous avons examiné l'impact de ce neuromodulateur sur les propriétés des neurones à bouffées [1]. Pour cela nous avons eu recours à l'optogénétique, une technique qui permet d'activer de façon sélective, grâce à

la lumière bleue, les neurones qui libèrent de la séro-

$(\rightarrow)$ Voir la Synthèse de M. Vandecasteele et al., $m / s n^{\circ} 4$, avril 2015, page 404 tonine [6] $(\rightarrow)$.

$\varepsilon$ n analysant une souris exprimant le canal à rhodopsine - canal cationique photoactivable - dans les neurones sérotoninergiques, nous avons montré que la libération de sérotonine dans la fente synaptique inhibait les décharges en bouffées enregistrées dans le subiculum (Figure 2, partie gauche). Des tests pharmacologiques et électrophysiologiques nous ont alors permis de montrer que la sérotonine se lie aux récepteurs $5-\mathrm{HT}_{2 \mathrm{C}}$ (5-hydroxytryptamine [serotonin] receptor $2 C$ ) qui, en activant une voie intracellulaire non encore identifiée, inhibent l'activation des canaux $\mathrm{Ca}_{\mathrm{V}} 3$ (Figure 2, partie droite). En conséquence, le nombre de potentiels d'action déclenchés au cours d'une bouffée diminue.

Nous avons ensuite testé si la voie modulatrice que nous avions découverte, pouvait avoir une influence sur les crises d'épilepsie. Pour cela, nous avons eu 
recours à deux modèles expérimentaux : des rats rendus épileptiques par une injection de pilocarpine, et des tranches de cerveau de souris devenues hyperexcitables par élimination des ions magnésium du milieu extracellulaire. Dans les deux cas, nous avons observé que la sérotonine induisait une forte diminution des décharges épileptiques (Figure 2). Nous avons obtenu des résultats similaires en appliquant des agonistes sélectifs pour les récepteurs $5-\mathrm{HT}_{2 \mathrm{C}}$ ou en bloquant les canaux calciques $\mathrm{Ca}_{\mathrm{v}}$.

\section{Applications cliniques}

Nos résultats suggèrent que la sérotonine empêche le déclenchement de décharges épileptiques, en agissant sur les récepteurs $5-\mathrm{HT}_{2 \mathrm{C}}$ des neurones principaux du subiculum. L'épilepsie est couramment traitée en diminuant l'excitabilité des neurones. Cet effet est obtenu soit en inhibant les canaux sodiques responsables des potentiels d'action, soit en augmentant l'activité des récepteurs inhibiteurs pour le GABA (l'acide gamma-aminobutyrique). Si cette approche soulage une majorité de patients, elle s'avère inefficace pour $30 \%$ d'entre eux. L'activation des récepteurs $5-\mathrm{HT}_{2 \mathrm{C}}$ représente une piste alternative à explorer. De nombreux cliniciens sont cependant réticents à cette perspective, car la sérotonine a la réputation de favoriser les crises d'épilepsie. Cette croyance provient du fait que de nombreux antidépresseurs favorisent les crises. Cependant, une revue systématique de la littérature montre que, seuls, les antidépresseurs tricycliques et tétracycliques induisent des convulsions. Ces molécules ne sont pas sélectives pour le système sérotoninergique, mais agissent également sur d'autres récepteurs comme ceux de l'adrénaline, de l'histamine ou de la muscarine. Les molécules sélectionnées pour le système sérotoninergique, telles que les inhibiteurs de la recapture de la sérotonine (SSRI), ne provoquent pas de crise $[7,8]$. Nos résultats suggèrent donc que l'activation des récepteurs $5-\mathrm{HT}_{2 \mathrm{C}}$ pourrait être bénéfique pour soigner l'épilepsie du lobe temporal. $\diamond$

Serotonin prevents temporall lobe epilepsy by inhibiting bursting neurons from the subiculum

\section{LIENS D'INTÉRÊT}

Les auteurs déclarent n'avoir aucun lien d'intérêt concernant les données publiées dans cet article.

\section{RÉFÉRENCES}

1. Petersen AV, Jensen CS, Crepel V, et al. Serotonin regulates the firing of principal cells of the subiculum by Inhibiting a T-type $\mathrm{Ca}^{2+}$ current. Front Cell Neurosci $2017 ; 11: 60$.

2. Cohen I, Navarro V, Clemenceau S, et al. On the origin of interictal activity in human temporal lobe epilepsy in vitro. Science $2002 ; 298: 1418-21$.

3. Menendez de la Prida L, Gal B. Synaptic contributions to focal and widespread spatiotemporal dynamics in the isolated rat subiculum in vitro. J Neurosci 2004 ; $24: 5525-36$

4. Harris $\varepsilon$, Stewart M. Intrinsic connectivity of the rat subiculum. II. Properties of synchronous spontaneous activity and a demonstration of multiple generator regions. J Comp Neurol 2001 ; 435 : 506-18.

5. Su H, Sochivko D, Becker A, et al. Upregulation of a T-type $\mathrm{Ca}^{2+}$ channel causes a long-lasting modification of neuronal firing mode after status epilepticus. J Neurosci $2002 ; 22$ : 3645-55.

6. Vandecasteele M, Senova YS, Stéphane Palfi S, et al. Potentiel thérapeutique de la neuromodulation optogénétique Med Sci (Paris) 2015 ; 31 : 404-16.

7. Montgomery SA. Antidepressants and seizures: emphasis on newer agents and clinical implications. Int J Clin Pract 2005 ; 59 : 1435-40.

8. Pisani F, Spina $\varepsilon$, Oteri G. Antidepressant drugs and seizure susceptibility: from in vitro data to clinical practice. Epilepsia 1999; 40 (suppl 10) : S48-56.

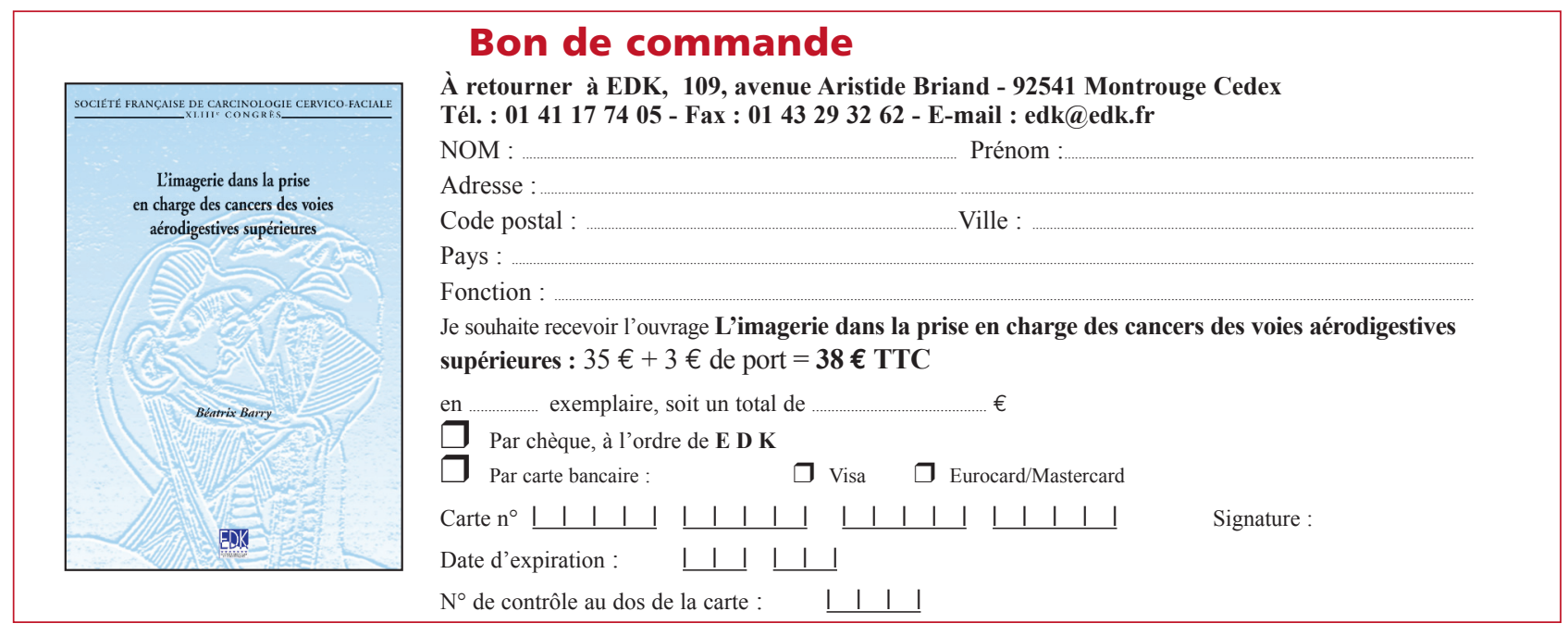

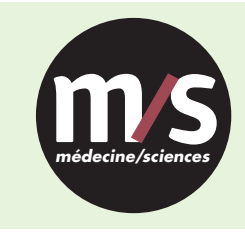

Tarifs d'abonnement $\mathrm{m} / \mathrm{s}-2017$

Abonnez-vous

à médecine/sciences
$>$ Grâce à $m / s$, vivez en direct les progrès des sciences biologiques et médicales

Bulletin d'abonnement page 806 dans ce numéro de $\mathrm{m} / \mathrm{s}$

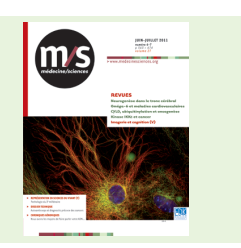

